\title{
Entangled radiation via a Raman-driven quantum-beat laser
}

\author{
Sajid Qamar, ${ }^{1}$ M. Al-Amri, ${ }^{2}$ Shahid Qamar, ${ }^{1, *}$ and M. Suhail Zubairy ${ }^{1,3}$ \\ ${ }^{1}$ Centre for Quantum Physics, COMSATS Institute of Information Technology, Islamabad 44000, Pakistan \\ ${ }^{2}$ The National Centre for Mathematics and Physics, KACST, P.O. Box 6086, Riyadh 11442, Saudi Arabia \\ ${ }^{3}$ Institute for Quantum Studies and Department of Physics, Texas A\&M University, College Station, Texas 77843-4242, USA
}

(Received 1 June 2009; published 10 September 2009)

\begin{abstract}
We propose a scheme for the entanglement generation of two cavity modes using a four-level Raman-driven quantum-beat laser (QBL). The atomic coherence is generated by a Raman-type two-photon process. Two different sufficient conditions for entanglement generation have been investigated. The effect of cavity losses and strength of the driving fields on entanglement generation is studied for different initial states of the resonant cavity modes. The results are in agreement with the earlier scheme for the entanglement generation using a directly driven quantum-beat laser. We observe entanglement generation even for high cavity losses and with low strength of the driving fields. Further, there is no dipole-forbidden transition in the Raman-driven QBL system. Thus the proposed scheme for the entanglement generation is experimentally more viable as compared to the previous directly driven QBL system.
\end{abstract}

DOI: $10.1103 /$ PhysRevA.80.033818

PACS number(s): 42.50.Pq, 03.67.Mn, 42.50.Md, 42.50.Ar

\section{INTRODUCTION}

Atomic coherence in a two-mode laser can generate fields that remained entangled for a certain period of interaction time. This has been investigated in a series of proposals for the generation of two-mode entangled radiation. Recently, two-photon correlated emission laser (CEL) has emerged as the most probable source of entangled radiation with a large number of photons in each mode [1]. The analysis has further been extended to observe continuous-variable entanglement [2], single atom as a macroscopic source of radiation entanglement [3], and to study the influence of pump-phase fluctuations on the entanglement generation [4]. In a parallel attempt, the quantum-beat laser (QBL) [5] has also been observed as a source of entangled radiation using two-level [6] and three-level [7] atomic systems.

Earlier, Polzik and co-workers demonstrated that quantum state transfer from nonclassical light to atom can be used for the realization of macroscopic entangled atomic ensemble [8]. Besides, several other proposals have been presented for the generation of entangled states of photons. These include the generation of two-mode quadrature squeezed light from a narrow-band nondegenerate optical parametric amplifier, polarization entangled radiation from parametric down conversion driven by intense pump field inside a cavity, and many others [9-15].

In the three-level CEL-based system the generation of entangled radiation depends on the coherent superposition of the upper and the lower atomic levels and the photons are emitted in cascade transitions, whereas in the three-level QBL-based system upper two atomic levels are prepared in a coherent superposition state and the photons are emitted in $V$ transitions. However, in both the systems one faces a common problem of dipole-forbidden transition. To avoid such a

\footnotetext{
*Permanent address: Department of Physics and Applied Mathematics, Pakistan Institute of Engineering and Applied Sciences, Nilore, Islamabad, Pakistan.
}

dipole-forbidden transition in a CEL-based system, we have proposed a scheme where upper and lower atomic levels are prepared in a coherent superposition state using a fourth Raman level [16]. We call this entanglement generation system as entanglement amplifier via Raman-driven coherence.

In this paper, we consider the entanglement generation of two cavity mode photons in a quantum-beat laser via Ramandriven coherence. That is, the two upper atomic levels are prepared in a coherent superposition of states using an auxiliary level. Here, the system consists of four-level atoms interacting with two modes of the cavity field in a doubly resonant cavity. The atoms are initially pumped incoherently to the auxiliary level and the coherent superposition state of the two excited levels is prepared using two strong driving fields via Raman-type two-photon process. The motivation comes from an earlier work by Rathe and Scully [17] where the role of phase coherence and decoherence has been investigated in the quantum-beat laser and in which the coherence has been generated via Raman-type two-photon process. Using this four-level system the experimental difficulties due to the presence of dipole-forbidden transition can be circumvented in the entanglement generation of the two cavity modes using a quantum-beat laser.

\section{ENTANGLEMENT CRITERIA}

We know that a system is entangled if it is not separable, i.e., the density matrix $\rho$ cannot be expressed as a convex combination of product states

$$
\rho=\sum_{j} p_{j} \rho_{j}^{(1)} \otimes \rho_{j}^{(2)},
$$

with $p_{j} \geq 0$ and $\Sigma_{j} p_{j}=1$. In this paper, we use the recently proposed Hillery-Zubairy (HZ) criterion [18] and SimonDuan-Giedke-Cirac-Zoller (DGCZ) criterion [19] to estimate the entanglement generation of the two modes of the cavity fields via a Raman-driven quantum-beat laser. These criteria provide a class of inequalities whose violation shows the presence of entanglement in a two-mode system. For general 
states, these are the sufficient conditions; however, for twomode continuous-variable Gaussian states the latter criterion becomes sufficient and necessary condition [2]. We consider different initial states of the two-mode fields and discuss the temporal evolution of the fields, which depends on the amplitudes of the Rabi frequencies associated with the two driving fields and the cavity decays.

The Hillery-Zubairy criterion states that

$$
\left\langle N_{1} N_{2}\right\rangle-\left|\left\langle a_{1} a_{2}^{\dagger}\right\rangle\right|^{2}<0
$$

where $N_{1}=a_{1}^{\dagger} a_{1}$ and $N_{2}=a_{2}^{\dagger} a_{2}$ are the photon number operators in modes 1 and 2 , respectively, whereas the SimonDGCZ criterion states that the system is known to be entangled if the quantum fluctuations of the two EinsteinPodolsky-Rosen-like operators, $\hat{u}$ and $\hat{v}$, of the two modes satisfy the following inequality:

$$
(\Delta \hat{u})^{2}+(\Delta \hat{v})^{2}<2 .
$$

Here,

$$
\begin{aligned}
& \hat{u}=\hat{x}_{1}+\hat{x}_{2}, \\
& \hat{v}=\hat{p}_{1}-\hat{p}_{2},
\end{aligned}
$$

and $x_{j}=\left(a_{j}+a_{j}^{\dagger}\right) / \sqrt{2}$ and $p_{j}=\left(a_{j}-a_{j}^{\dagger}\right) / \sqrt{2} i$ (with $j=1,2$ ) are the quadratures for the two modes of the cavity field. These can be measured in an experiment by using the technique of balanced homodyne detection [20]. On substituting the definition of $\hat{u}$ and $\hat{v}$ in Eq. (3), we obtain

$$
\begin{aligned}
(\Delta \hat{u})^{2}+(\Delta \hat{v})^{2}= & \left\langle a_{1}^{\dagger}, a_{1}\right\rangle+\left\langle a_{1}, a_{1}^{\dagger}\right\rangle+\left\langle a_{2}^{\dagger}, a_{2}\right\rangle+\left\langle a_{2}, a_{2}^{\dagger}\right\rangle \\
& +2\left(\left\langle a_{1}, a_{2}\right\rangle+\left\langle a_{1}^{\dagger}, a_{2}^{\dagger}\right\rangle\right),
\end{aligned}
$$

where we use the notation $\langle a, b\rangle=\langle a b\rangle-\langle a\rangle\langle b\rangle$. In order to study the entanglement, we calculate the time evolution of various moments involved in the inequalities in Eqs. (2) and (3) using the equations of motion given in Appendix A.

\section{MODEL}

We consider a four-level Raman-driven quantum-beat laser system as shown in Fig. 1. The atoms which are pumped in the auxiliary state $|c\rangle$ at a rate $r_{c}$ interact with two modes $\nu_{1}$ and $\nu_{2}$ of the field inside the doubly resonant cavity. The corresponding transitions are $\left|b_{1}\right\rangle \leftrightarrow|a\rangle$ and $\left|b_{2}\right\rangle \leftrightarrow|a\rangle$. The coherence between levels $\left|b_{1}\right\rangle$ and $\left|b_{2}\right\rangle$ is generated using two strong classical driving fields via a fourth level $|c\rangle$ in a Raman-type two-photon process. The corresponding Rabi frequencies are $\Omega_{1}$ and $\Omega_{2}$. We also consider that all atomic levels decay at the same rate $\Gamma$ to some other atomic levels outside the system. Such a system has been discussed within the context of phase coherence and decoherence in the quantum-beat laser by Rathe and Scully [17]. The interaction picture Hamiltonian for this system is given by

$$
\begin{aligned}
V= & \hbar g\left(a_{1} e^{i \Delta_{1} t}\left|b_{1}\right\rangle\left\langle a\left|+a_{2} e^{i \Delta_{2} t}\right| b_{2}\right\rangle\langle a|\right)-\frac{\hbar \Omega_{1}}{2}|c\rangle\left\langle b_{1}\left|-\frac{\hbar \Omega_{2}}{2}\right| c\right\rangle \\
& \times\left\langle b_{2}\right|+\text { H.a. }
\end{aligned}
$$

Here, the detuning $\Delta_{1}\left(\Delta_{2}\right)$ is defined as $\Delta_{1}=\omega_{b_{1} a}-\nu_{1}\left(\Delta_{2}\right.$

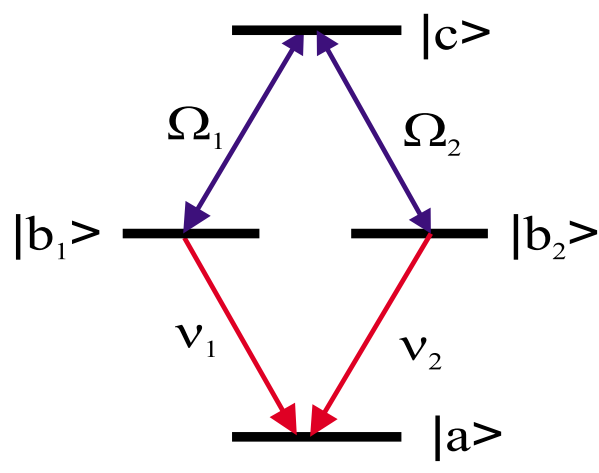

FIG. 1. (Color online) Energy-level diagram of a four-level Raman-driven quantum-beat laser. The transition between levels $\left|b_{1}\right\rangle-|a\rangle$ and $\left|b_{2}\right\rangle-|a\rangle$ at frequencies $\nu_{1}$ and $\nu_{2}$ are resonant with the cavity. Two strong driving fields are applied between levels $|c\rangle \leftrightarrow\left|b_{1}\right\rangle$ and $|c\rangle \leftrightarrow\left|b_{2}\right\rangle$. The corresponding Rabi frequencies are $\Omega_{1}$ and $\Omega_{2}$, respectively.

$\left.=\omega_{b_{2} a}-\nu_{2}\right), a_{1}\left(a_{1}^{\dagger}\right)$ and $a_{2}\left(a_{2}^{\dagger}\right)$ are the annihilation (creation) operators of the cavity fields, whereas $g$ is the coupling constant associated with $\left|b_{1}\right\rangle$ and $\left|b_{2}\right\rangle$ to $|a\rangle$ transitions.

The equation of motion for the field density matrix is given by

$$
\begin{aligned}
\dot{\rho}_{f}= & -1 / 2\left[\alpha_{11}^{*} a_{1} a_{1}^{\dagger} \rho_{f}+\alpha_{11} \rho_{f} a_{1} a_{1}^{\dagger}-\left(\alpha_{11}+\alpha_{11}^{*}\right) a_{1}^{\dagger} \rho_{f} a_{1}\right] \\
& -1 / 2\left[\alpha_{22}^{*} a_{2} a_{2}^{\dagger} \rho_{f}+\alpha_{22} \rho_{f} a_{2} a_{2}^{\dagger}-\left(\alpha_{22}+\alpha_{22}^{*}\right) a_{2}^{\dagger} \rho_{f} a_{2}\right] \\
& -1 / 2\left[\alpha_{21}^{*} a_{1}^{\dagger} a_{2} \rho_{f}+\alpha_{12} \rho_{f} a_{1}^{\dagger} a_{2}-\left(\alpha_{12}+\alpha_{21}^{*}\right) a_{1}^{\dagger} \rho_{f} a_{2}\right] e^{i \Phi} \\
& -1 / 2\left[\alpha_{12}^{*} a_{1} a_{2}^{\dagger} \rho_{f}+\alpha_{21} \rho_{f} a_{1} a_{2}^{\dagger}-\left(\alpha_{21}+\alpha_{12}^{*}\right) a_{2}^{\dagger} \rho_{f} a_{1}\right] e^{-i \Phi} \\
& -\kappa_{1}\left(a_{1}^{\dagger} a_{1} \rho_{f}-2 a_{1} \rho_{f} a_{1}^{\dagger}+\rho_{f} a_{1}^{\dagger} a_{1}\right)-\kappa_{2}\left(a_{2}^{\dagger} a_{2} \rho_{f}-2 a_{2} \rho_{f} a_{2}^{\dagger}\right. \\
& \left.+\rho_{f} a_{2}^{\dagger} a_{2}\right),
\end{aligned}
$$

where we include the cavity damping terms in the usual way, assuming that the two cavity modes are coupled to two independent vacuum reservoirs with $\kappa_{1}$ and $\kappa_{2}$ being the cavity decay rates of modes 1 and 2, respectively. Here, $\alpha_{11}$ and $\alpha_{22}$ are the linear gain coefficients, whereas $\alpha_{12}$ and $\alpha_{21}$ are the phase-sensitive cross-coupling coefficients that are generated due to the atomic coherence produced between the levels $\left|b_{1}\right\rangle$ and $\left|b_{2}\right\rangle$ via the two-photon Raman process using two strong driving fields. Explicit expressions for the coefficients $\alpha_{11}$, $\alpha_{12}, \alpha_{21}$, and $\alpha_{22}$ in Eq. (7) are given in Appendix B. The phase angle $\Phi$ appearing in Eq. (7) equals $\Phi=\phi+\left(\nu_{1}-\nu_{2}\right.$ $\left.-\omega_{b_{1} b_{2}}\right) t$ with $\phi$ being the phase associated with the driving fields.

\section{DISCUSSION}

We know that the transverse decay decreases the degree of coherence irrespective of the strength of the driving fields; therefore, in our scheme these decays are considered to be zero. In this situation the ideal CEL action occurs without assumptions about the strength of the driving fields [17]. However, the threshold limits still prevail for the entanglement generation in the system and for different choices of the 
Rabi frequencies of the driving fields and cavity decay rates, we expect an increase or a decrease in the entanglement generation.

For simplicity's sake, we consider $\Delta=\Delta_{1}=\Delta_{2}$ and $\Omega_{1}$ $=\Omega_{2}=\Omega$. Therefore, all the gain and the cross-coupling coefficients defined in Appendix B become equal and can be written as

$$
\alpha=\frac{2 r_{c} g^{2}}{D}\left\{\frac{\Omega^{2}\left(\Omega^{2}+2 \Gamma^{2}\right)(3 \Gamma+2 i \Delta)}{\left(\Omega^{2}-2 \Delta^{2}+2 \Gamma^{2}+4 i \Delta \Gamma\right)}\right\},
$$

where $\alpha=\alpha_{11}=\alpha_{22}=\alpha_{12}=\alpha_{21}$ and

$$
D=\Gamma\left(4 \Gamma^{4}+10 \Omega^{2} \Gamma^{2}+4 \Omega^{4}\right) .
$$

In recent studies, we have discussed the generation of entanglement in a parametric converter [15] and quantumbeat laser [7] using the Simon-DGCZ and the HilleryZubairy criteria and obtained the conditions on the input modes that ensure that the output modes are entangled. We know from these studies that the entanglement generation via a quantum-beat laser strongly depends on the strength of the driving fields and the cavity decay rates. In this proposal we investigate the entanglement generation via a Raman-driven quantum-beat laser and study the influence of cavity decay rates and strength of the driving fields on it. We note from the linear gain and cross-coupling coefficients that there is no parametric limit exists where the Raman-driven quantumbeat laser becomes a parametric converter [7,15]. This is the same situation observed in earlier studies of the CEL-based entanglement generation and phase-sensitive amplifier using Raman-driven coherence [16,21]. Therefore, there exists minimum and maximum threshold values for the driving field Rabi frequencies for which the entanglement between two cavity modes is generated.

We would also like to mention that exact analytical results in this proposal are rather complicated, and therefore in the following we shall only present the numerical results and analysis. The system we discuss here can be realized by placing the gain medium inside a doubly resonant cavity. We choose the parameter values which correspond to the micromaser experiment [27]. The second alternative experimental approach may be a system in which atoms with long-lived states pass through the doubly resonant cavity such that only one atom is inside the cavity at a time which corresponds to the micromaser experiment carried out by several groups $[27,28]$. In the following analysis, we consider exact resonance $\left(\Delta=\Delta_{1}=\Delta_{2}=0\right)$ under the condition $\Phi=0$.

We start our analysis with the initial coherent state having $\alpha=10$ in mode 1 and squeezed vacuum state in mode 2 . The squeezed vacuum state can be represented as $S(r)|0\rangle$ with the squeezing operator $S(r)=\exp \left[(r / 2)\left(a^{2}-a^{\dagger^{2}}\right)\right]$ defined in terms of the creation and the annihilation operators $a$ and $a^{\dagger}$ with squeezing parameter $r=0.1$ and photon number $n=0$. Figure 2 shows the time evolution of the $\mathrm{HZ}$ criterion $F_{1}$ $=\left\langle N_{1} N_{2}\right\rangle-\left|\left\langle a_{1} a_{2}^{\dagger}\right\rangle\right|^{2}$ versus the dimensionless interaction time $\beta$ t. The parameter $\beta$ is defined as $\beta=2 g^{2} r_{c} / \Gamma^{2}$ and other corresponding parameters are given in the figure caption. For simplicity in analysis we consider $\Omega_{1}=\Omega_{2}=\Omega$ and $\kappa_{1}=\kappa_{2}$ $=\kappa$. We observe that $F_{1}$ becomes negative as time evolves,

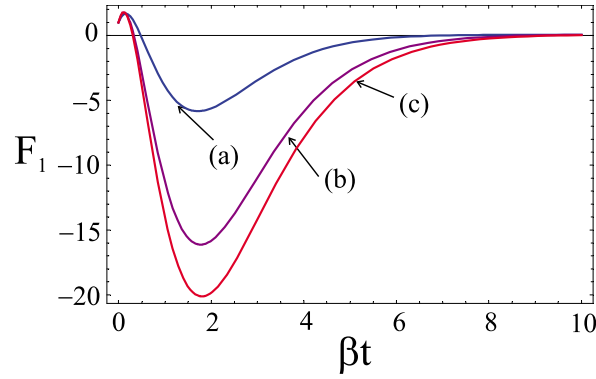

FIG. 2. (Color online) Influence of the Rabi frequencies on the entanglement generation; time evolution of $F_{1}=\left\langle N_{1} N_{2}\right\rangle-\left|\left\langle a_{1} a_{2}^{\dagger}\right\rangle\right|^{2}$ against the normalized interaction time $\beta t$ for initial squeezed vacuum state in mode 1 with $r=0.1$ and initial coherent state in mode 2 with $\alpha=10$. Other parameters are $\Gamma=20 \mathrm{kHz}, \kappa_{1}=\kappa_{2}$ $=5 \mathrm{kHz}, g=16 \mathrm{kHz}$, and $r_{c}=10 \mathrm{kHz}$. The strength of the driving fields corresponds to $\Omega_{1}=\Omega_{2}=\Omega$ equals (a) 10 , (b) 15 , and (c) 20 $\mathrm{kHz}$.

which shows that the two cavity modes, which are initially in the product state, evolve into entangled state. The two modes are remained entangled for a certain duration of interaction time $\beta t$ and we observe that the entanglement strongly depends on the strength of the driving fields and the cavity losses.

The time evolution of $F_{1}$ in Fig. 2 exhibits the influence of the driving field strengths on the entanglement generation. Three different values of the corresponding Rabi frequencies are considered, i.e., $\Omega$ is equal to (a) 10 , (b) 15 , and (c) 20 $\mathrm{kHz}$. The quantity $F_{1}$ becomes negative after a small interaction time $\beta t$, which implies that the two cavity modes at that particular interaction time evolve into an entangled state. The quantity $F_{1}$ remained negative for a certain interval of time, and therefore the entanglement is sustained for that duration. Here, we note the strong dependence of entanglement on the strength of the driving fields. That is, for three different choices of the Rabi frequencies corresponding to the driving fields, we get three different time intervals for the entanglement between the two cavity modes. We observe that the time interval for the entanglement increases with an increase in the Rabi frequencies of the driving fields. This is due to the increase in coherence between the two levels $\left|b_{1}\right\rangle$ and $\left|b_{2}\right\rangle$ via level $|c\rangle$. These results are in agreement with our earlier study of entanglement generation in the quantum-beat laser [7] except that in the present case we do not observe oscillations in $F_{1}$. The oscillations in $F_{1}$ in the directly driven quantum-beat laser system for the entanglement generation also vanish when relatively high strength of the driving field is considered (see Fig. 2 in Ref. [7]).

To see the mean photon number behavior in the two cavity modes during the time of entanglement, we plot the time evolution of $\left\langle N_{1}\right\rangle$ and $\left\langle N_{2}\right\rangle$ versus $\beta t$ in Fig. 3. We consider the same values of the corresponding parameters given in the case of Fig. 2. The main plot reflects the time evolution of the mean photon number in mode 1 and the inset gives the mean photon number evolution in mode 2 . We observe that the mean number of photons in mode $1\left\langle N_{1}\right\rangle$, which is 100 photons at time $\beta t=0$, decreases almost exponentially. The inset of Fig. 3 shows the time evolution of $\left\langle N_{2}\right\rangle$ where initially the mean number of photons is zero. It increases to 


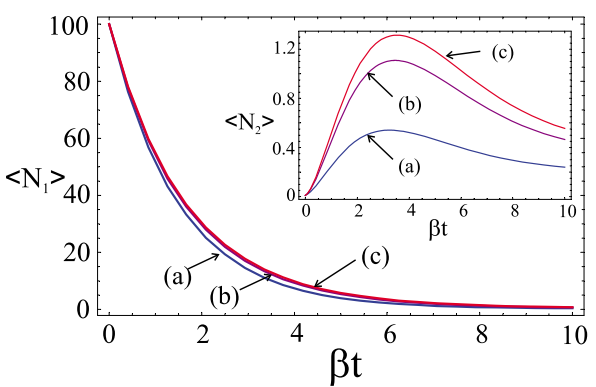

FIG. 3. (Color online) Photon number evolution during entanglement; time evolution of $\left\langle N_{1}\right\rangle$ (main plot) and $\left\langle N_{2}\right\rangle$ (inset) against the normalized interaction time $\beta t$ for three different driving fields. The parameters are the same as in Fig. 2.

some maximum value and than decreases with time $\beta t$ to a steady-state value. We note that the behavior of $\left\langle N_{1}\right\rangle$ remains almost unaffected by the strength of the driving fields. However, the inset in Fig. 3 shows that the mean photon number in mode 2 depends on the strength of the driving fields. We also notice that the mean photon number $\left\langle N_{1}\right\rangle$ does not show an oscillatory behavior as has been observed in the earlier studies [7]. This conforms with our initial observation of no oscillatory behavior in the quantity $F_{1}$ (see Fig. 2).

To see the effect of cavity losses on the entanglement generation, we plot $F_{1}$ versus $\beta t$ for three different values of the cavity decay rates (see Fig. 4). We observe that as the cavity decay rates decrease the quantity $F_{1}$ becomes more negative and the time during which the entanglement is generated increases considerably. This is according to the physical behavior of the entanglement generation system, i.e., cavity losses disturb the threshold conditions for the entanglement generation and consequently the entanglement vanishes for high enough cavity decay rates. These results are also in agreement with earlier studies of entanglement generation via the parametric converter [15]. From Figs. 2 and 4, we further note that in this Raman-driven QBL scheme the entanglement between two cavity modes is generated even for high cavity losses and low strength of the driving fields.

Next, we change the initial states of the two cavity modes and consider that mode 1 is initially in the vacuum state and mode 2 is in the photon number state with ten photons, i.e., $n=10$. The quantity $F_{1}$ becomes negative in this case as well

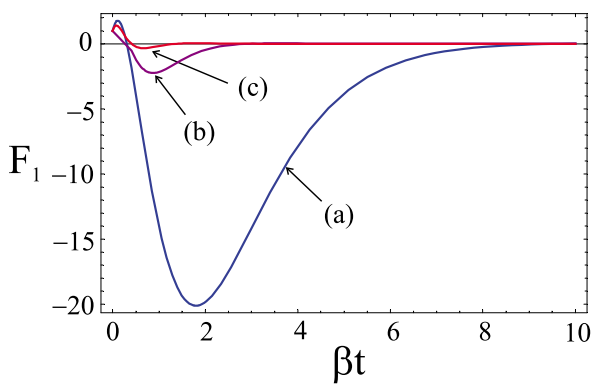

FIG. 4. (Color online) Influence of the cavity losses on the entanglement generation; time evolution of $F_{1}$ versus $\beta t$ for $\Omega_{1}=\Omega_{2}$ $=\Omega=20 \mathrm{kHz}$ and the cavity decay rates $\kappa_{1}=\kappa_{2}=\kappa$ are (a) 5, (b) 10 , and (c) $15 \mathrm{kHz}$. The other parameters are the same as in Fig. 2.

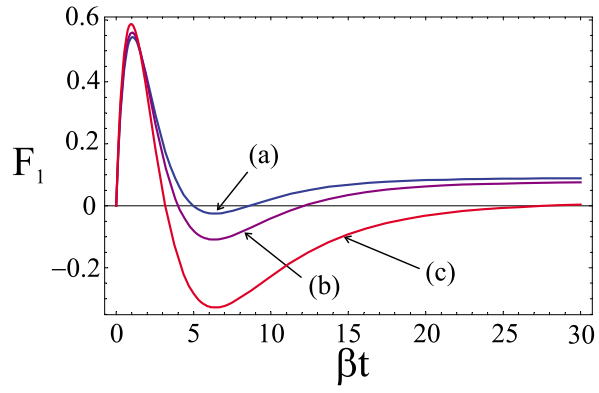

FIG. 5. (Color online) Influence of the Rabi frequencies on the entanglement generation; time evolution of $F_{1}$ versus $\beta t$ for initial vacuum state in mode 1 and photon number state in mode 2 with $n=10$. The cavity losses correspond to $\kappa_{1}=\kappa_{2}=3 \mathrm{kHz}$ and Rabi frequencies are (a) 12, (b) 13, and (c) $15 \mathrm{kHz}$. Other parameters are the same as in Fig. 2.

which shows that the entangled radiation of the two cavity modes is generated. Here, we again observe the strong dependence of the entanglement generation on the strength of the driving fields. Therefore, the entanglement between two modes increases with an increase in the Rabi frequencies of the driving fields (see the behavior of $F_{1}$ in Fig. 5). This shows that the entanglement in the Raman-driven QBL can be generated with different initial states of the cavity modes. The influence of the cavity losses on the entanglement generation is again studied in Fig. 6, which reflects a similar behavior observed in Fig. 4.

As a second choice, we consider the Simon-DGCZ criterion to study the entanglement generation in the proposed system. We plot $F_{2}=(\Delta \hat{u})^{2}+(\Delta \hat{v})^{2}$ versus $\beta t$, which is shown in Fig. 7. We consider three different Rabi frequencies, i.e., $\Omega$ equals (a) 6000, (b) 5000, and $4500 \mathrm{kHz}$. The dependence of the entanglement generation on the Rabi frequencies again shows that the duration of entanglement increases with an increase in the strength of the driving fields, which is again in accordance with the earlier studies $[7,16]$.

In Fig. 8, we plot the Simon-DGCZ criterion versus $\beta t$ for three different values of the cavity decay rates, i.e., $\kappa$ equals (a) 1.5 , (b) 3.0 , and (c) $6.0 \mathrm{kHz}$. It is obvious from the figure that the Simon-DGCZ criterion also establishes strong dependence of the entanglement generation on the cavity losses. As the cavity decay rates increases, the duration of

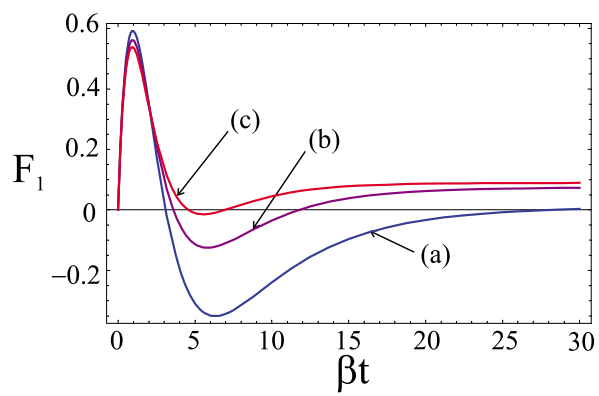

FIG. 6. (Color online) Influence of the cavity losses on the entanglement generation; time evolution of $F_{1}$ versus $\beta t$ for $\Omega_{1}=\Omega_{2}$ $=15 \mathrm{kHz}$ and cavity decay rates $\kappa_{1}=\kappa_{2}=\kappa$ are (a) 3, (b) 3.25 , and (c) $3.5 \mathrm{kHz}$. The other parameters are the same as in Fig. 5. 


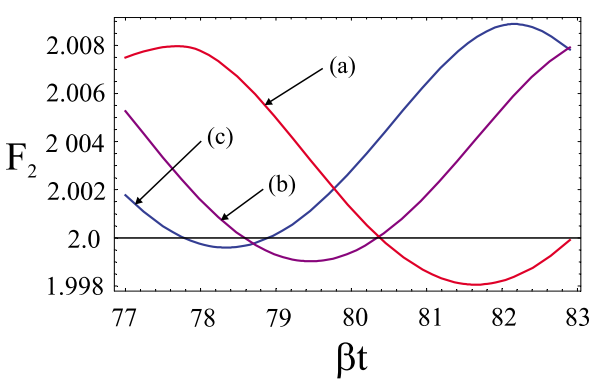

FIG. 7. (Color online) Time evolution of $F_{2}=(\Delta \hat{u})^{2}+(\Delta \hat{v})^{2}$ versus $\beta t$; influence of Rabi frequencies on the entanglement. We consider initial squeezed vacuum state with $r=0.1$ in mode 1 and coherent state in mode 2 with $\alpha=200$, whereas $g=43 \mathrm{kHz}$ and $\kappa_{1}$ $=\kappa_{2}=1.5 \mathrm{kHz}$. Three choices of the Rabi frequencies are (a) 6000, (b) 5000 , and $4500 \mathrm{kHz}$.

entanglement decreases and eventually for higher cavity losses entanglement vanishes completely.

We would like to mention here that we have considered much higher values of the Rabi frequencies for the SimonDGCZ criterion as compared to the HZ criterion. In both cases the time evolution exhibits entangled radiation whenever the threshold condition for the entanglement is reached. This reflects that both criteria are the sufficient and not necessary conditions for the entanglement generation. However, it is also possible to observe the entanglement between the two cavity modes using both criteria for the same choices of parameters. Therefore, in the last plot (Fig. 9), we show the time evolutions of the Simon-DGCZ and the HZ criteria when $\Omega=4500 \mathrm{kHz}$ while keeping the rest of the parameters as in Fig. 8. This gives a comparison of the entanglement generation via the $\mathrm{HZ}$ criterion (main plot) and the SimonDGCZ criterion (inset). We find that the two criteria give different time intervals for the entanglement generation of the two modes for the same values of the parameters. This satisfies the earlier studies that both criteria give only sufficient condition for the entanglement generation of the two modes.

\section{CONCLUSION}

We have investigated the entanglement generation of the two cavity modes using a Raman-driven quantum-beat laser.

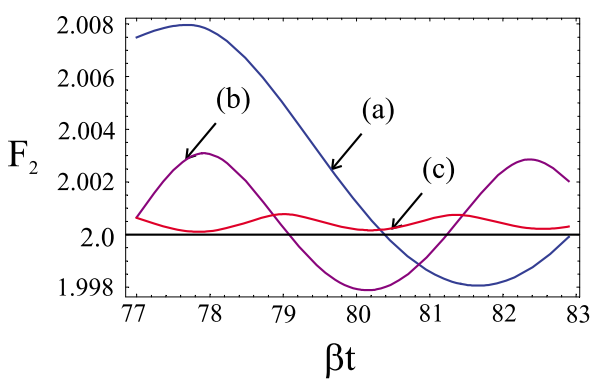

FIG. 8. (Color online) Time evolution of $F_{2}$ versus $\beta t$; influence of cavity losses on the entanglement. We consider $\Omega_{1}=\Omega_{2}$ $=6000 \mathrm{kHz}$ and the cavity decay rates are (a) 1.5, (b) 3.0, and (c) $6.0 \mathrm{kHz}$.

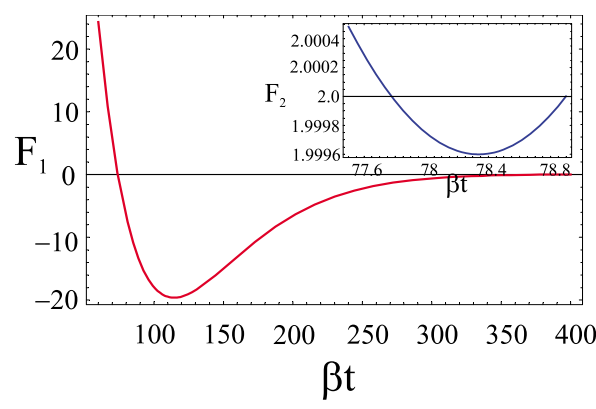

FIG. 9. (Color online) Time evolution of $F_{1}$ (main plot) and $F_{2}$ (inset) versus $\beta t$ for $\Omega=4500 \mathrm{kHz}$. The other parameters are the same as in Fig. 7.

The coherence in the upper two levels of the QBL is generated via two-photon Raman process using an auxiliary level which avoids the use of dipole-forbidden transition and an ideal CEL action is possible without the need for strong driving fields [17]. Two different entanglement criteria, i.e., Hillery-Zubairy and Simon-DGCZ criteria, are used to study the entangled radiation for different initial states of the cavity modes and different sets of parameters. We also considered that the transverse decay (due to the collisions between lasing atoms) is zero. This may lead to an ideal CEL action without assumptions about the strength of the driving fields.

The time evolution of the HZ criterion exhibits that the Raman-driven QBL becomes a source of entangled radiation even for low strength of the driving fields and high cavity losses. However, the duration of the entanglement generation changes with the strength of the driving fields and cavity losses. The time evolution of the photon statistics is also plotted and we observed no oscillation in the mean number photon behavior of the two cavity modes. We have noticed that the Raman-driven QBL system becomes a source of entangled radiation for different initial states of the cavity modes; however, the threshold values for the entangled radiation are different for different sets of parameters.

The time evolution of the Simon-DGCZ criterion is also plotted for different strengths of the driving fields and cavity losses. We observed that the duration of the entanglement generation again depends on the strength of the driving fields and cavity losses. However, in this case we have considered much higher values of the driving filed Rabi frequencies. Here, we would like to mention that the two criteria ( $\mathrm{HZ}$ and Simon-DGCZ) are only sufficient conditions for the entanglement generation. For a comparison we plotted the time evolutions of the two entanglement criteria for the same choice of parameters. We observed different initial times and intervals for the entanglement generation, which emphasizes the fact that both HZ and Simon-DGCZ criteria are the sufficient but not the necessary conditions for the entanglement. The mean number of photons in both cavity modes are also plotted to see some insight of the photon statistics. We also noticed from the linear gain and cross-coupling coefficients that the Raman-driven QBL system does not become a parametric converter under the parametric limits.

As concluding remarks we would like to mention that entanglement is essential in quantum-information processes such as quantum teleportation [22], quantum dense coding 
[23], quantum cryptography [24], and quantum computing [25]. In any quantum-information process, it is important to know whether a state is entangled or not before any application. It may be pointed out that continuous-variable entanglement can offer an advantage in some situations in quantum-information theory [26]. Our proposed Ramandriven quantum-beat laser system seems to be a good choice of the entanglement generation source as this is experimentally a more viable system $[17,27,28]$.

\section{ACKNOWLEDGMENTS}

The authors would like to thank COMSTECH for the support. This research was partially supported by grants from King Abdulaziz City for Science and Technology (KACST) and the Qatar National Research Fund (QNRF).

\section{APPENDIX A}

The equations of motion for various moments required to study the generation of entanglement using a Raman-driven quantum-beat laser can easily be obtained from Eq. (7). For a suitable choice of the phase $\Phi=0$ associated with the classical driving fields, the following sets of coupled equations for various moments are obtained:

$$
\begin{aligned}
& \frac{d}{d t}\left\langle a_{1}\right\rangle=\frac{1}{2}\left(\alpha_{11}-2 \kappa_{1}\right)\left\langle a_{1}\right\rangle+\frac{1}{2} \alpha_{12}\left\langle a_{2}\right\rangle \\
& \frac{d}{d t}\left\langle a_{2}\right\rangle=\frac{1}{2}\left(\alpha_{22}-2 \kappa_{2}\right)\left\langle a_{2}\right\rangle+\frac{1}{2} \alpha_{21}\left\langle a_{1}\right\rangle \\
\frac{d}{d t}\left\langle a_{1} a_{1}\right\rangle=\left(\alpha_{11}-2 \kappa_{1}\right)\left\langle a_{1} a_{1}\right\rangle+\alpha_{12}\left\langle a_{1} a_{2}\right\rangle & \\
\frac{d}{d t}\left\langle a_{2} a_{2}\right\rangle=\left(\alpha_{22}-2 \kappa_{2}\right)\left\langle a_{2} a_{2}\right\rangle+\alpha_{21}\left\langle a_{1} a_{2}\right\rangle & \\
\frac{d}{d t}\left\langle a_{1} a_{2}\right\rangle= & {\left[\frac{1}{2}\left(\alpha_{11}+\alpha_{22}\right)-\left(\kappa_{1}+\kappa_{2}\right)\right]\left\langle a_{1} a_{2}\right\rangle+\frac{1}{2} \alpha_{12}\left\langle a_{2} a_{2}\right\rangle } \\
& +\frac{1}{2} \alpha_{21}\left\langle a_{1} a_{1}\right\rangle, \\
& \left.+\alpha_{12}^{*}\left\langle a_{2}^{\dagger} a_{2}\right\rangle\right)+\frac{1}{2}\left(\alpha_{21}^{*}+\alpha_{12}\right) \\
\frac{d}{d t}\left\langle a_{1}^{\dagger} a_{2}\right\rangle= & \left.\left.\left.\frac{1}{2}\left[\left(\alpha_{11}^{*}+\alpha_{22}^{\dagger}\right)-\left(\kappa_{1}+a_{2}\right\rangle\right)+\frac{1}{2}\left(\alpha_{21}+\alpha_{12}^{*}\right\rangle\right)\right]\left\langle a_{1}^{\dagger}\right\rangle a_{2}\right\rangle+\frac{1}{2}\left(\alpha_{21}\left\langle a_{1}^{\dagger} a_{1}\right\rangle\right. \\
& \frac{1}{2}\left[\left(\alpha_{11}+\alpha_{22}^{*}\right)-\left(\kappa_{1}+\kappa_{2}\right)\right]\left\langle a_{1} a_{2}^{\dagger}\right\rangle+\frac{1}{2}\left(\alpha_{21}^{*}\left\langle a_{1}^{\dagger} a_{1}\right\rangle\right.
\end{aligned}
$$

$$
\begin{aligned}
\frac{d}{d t}\left\langle N_{1}\right\rangle= & \frac{1}{2}\left[\left(\alpha_{11}+\alpha_{11}^{*}\right)-2 \kappa_{1}\right]\left\langle N_{1}\right\rangle+\frac{1}{2}\left(\alpha_{12}\left\langle a_{1}^{\dagger} a_{2}\right\rangle\right. \\
& \left.+\alpha_{12}^{*}\left\langle a_{1} a_{2}^{\dagger}\right\rangle\right)+\frac{1}{2}\left(\alpha_{11}+\alpha_{11}^{*}\right), \\
\frac{d}{d t}\left\langle N_{2}\right\rangle= & \frac{1}{2}\left[\left(\alpha_{22}+\alpha_{22}^{*}\right)-2 \kappa_{2}\right]\left\langle N_{2}\right\rangle+\frac{1}{2}\left(\alpha_{21}^{*}\left\langle a_{1}^{\dagger} a_{2}\right\rangle\right. \\
& \left.+\alpha_{21}\left\langle a_{1} a_{2}^{\dagger}\right\rangle\right)+\frac{1}{2}\left(\alpha_{22}+\alpha_{22}^{*}\right),
\end{aligned}
$$

$$
\begin{aligned}
\frac{d}{d t}\left\langle N_{1} N_{2}\right\rangle= & {\left[\frac{1}{2}\left(\alpha_{11}+\alpha_{11}^{*}+\alpha_{22}+\alpha_{22}^{*}\right)-2\left(\kappa_{1}+\kappa_{2}\right)\right]\left\langle N_{1} N_{2}\right\rangle } \\
& +\frac{1}{2}\left(\alpha_{22}+\alpha_{22}^{*}\right)\left\langle N_{1}\right\rangle+\frac{1}{2}\left(\alpha_{11}+\alpha_{11}^{*}\right)\left\langle N_{2}\right\rangle \\
& +\frac{1}{2}\left(\alpha_{21}\left\langle N_{1} a_{1} a_{2}^{\dagger}\right\rangle+\alpha_{21}^{*}\left\langle N_{1} a_{1}^{\dagger} a_{2}\right\rangle\right) \\
& +\frac{1}{2}\left(\alpha_{12}^{*}\left\langle a_{1} a_{2}^{\dagger} N_{2}\right\rangle+\alpha_{12}\left\langle a_{1}^{\dagger} a_{2} N_{2}\right\rangle\right)+\frac{1}{2}\left(\alpha_{12}^{*}+\alpha_{21}\right) \\
& \times\left\langle a_{1} a_{2}^{\dagger}\right\rangle,
\end{aligned}
$$

$$
\begin{aligned}
\frac{d}{d t}\left\langle N_{1} a_{1}^{\dagger} a_{2}\right\rangle= & {\left[\frac{1}{2}\left(2 \alpha_{11}^{*}+\alpha_{11}+\alpha_{22}\right)-\left(3 \kappa_{1}+\kappa_{2}\right)\right]\left\langle N_{1} a_{1}^{\dagger} a_{2}\right\rangle } \\
& +\left[\frac{1}{2}\left(\alpha_{11}+\alpha_{11}^{*}\right)+2 \kappa_{1}\right]\left\langle a_{1}^{\dagger} a_{2}\right\rangle+\frac{1}{2} \alpha_{12}\left\langle a_{1}^{\dagger 2} a_{2}^{2}\right\rangle \\
& +\left[\alpha_{12}^{*}\left\langle N_{1} N_{2}\right\rangle+\frac{1}{2} \alpha_{21}\left\langle N_{1}^{2}\right\rangle+\frac{1}{2}\left(\alpha_{12}^{*}+\alpha_{21}\right)\left\langle N_{1}\right\rangle\right. \\
& \left.+\frac{1}{2} \alpha_{12}^{*}\left\langle N_{2}\right\rangle+\frac{1}{2}\left(\alpha_{12}^{*}+\alpha_{21}\right)\right],
\end{aligned}
$$

$$
\begin{aligned}
\frac{d}{d t}\left\langle N_{1} a_{1} a_{2}^{\dagger}\right\rangle= & {\left[\frac{1}{2}\left(\alpha_{11}^{*}+2 \alpha_{11}+\alpha_{22}^{*}\right)-\left(3 \kappa_{1}+\kappa_{2}\right)\right]\left\langle N_{1} a_{1} a_{2}^{\dagger}\right\rangle } \\
& +\left(\alpha_{11}+\alpha_{11}^{*}\right)\left\langle a_{1} a_{2}^{\dagger}\right\rangle+\frac{1}{2} \alpha_{12}^{*}\left\langle a_{1}^{2} a_{2}^{\dagger}\right\rangle \\
& -\left[\alpha_{12}\left\langle N_{1} N_{2}\right\rangle+\frac{1}{2} \alpha_{21}^{*}\left\langle N_{1}^{2}\right\rangle+\frac{1}{2}\left(2 \alpha_{12}+\alpha_{21}^{*}\right)\right. \\
& \left.\times\left\langle N_{1}\right\rangle\right],
\end{aligned}
$$$$
\frac{d}{d t}\left\langle a_{1} a_{2}^{\dagger} N_{2}\right\rangle=\left[\frac{1}{2}\left(\alpha_{11}+2 \alpha_{22}^{*}+\alpha_{22}\right)-\left(\kappa_{1}+3 \kappa_{2}\right)\right]\left\langle a_{1} a_{2}^{\dagger} N_{2}\right\rangle
$$$$
+\left(\alpha_{22}+\alpha_{22}^{*}\right)\left\langle a_{1} a_{2}^{\dagger}\right\rangle+\frac{1}{2} \alpha_{21}\left\langle a_{1}^{2} a_{2}^{\dagger 2}\right\rangle
$$$$
+\left[\alpha_{21}^{*}\left\langle N_{1} N_{2}\right\rangle+\frac{1}{2} \alpha_{12}\left\langle N_{2}^{2}\right\rangle+\frac{1}{2}\left(2 \alpha_{21}^{*}+\alpha_{12}\right)\right.
$$$$
\left.\times\left\langle N_{2}\right\rangle\right]
$$ 


$$
\begin{aligned}
& \frac{d}{d t}\left\langle a_{1}^{\dagger} a_{2} N_{2}\right\rangle=\left[\frac{1}{2}\left(\alpha_{11}^{*}+\alpha_{22}^{*}+2 \alpha_{22}\right)-\left(\kappa_{1}+3 \kappa_{2}\right)\right]\left\langle a_{1}^{\dagger} a_{2} N_{2}\right\rangle \\
& +\left[\frac{1}{2}\left(\alpha_{22}+\alpha_{22}^{*}\right)+2 \kappa_{2}\right]\left\langle a_{1}^{\dagger} a_{2}\right\rangle+\frac{1}{2} \alpha_{21}^{*}\left\langle a_{1}^{\dagger 2} a_{2}^{2}\right\rangle \\
& +\left[\alpha_{21}\left\langle N_{1} N_{2}\right\rangle+\frac{1}{2} \alpha_{12}^{*}\left\langle N_{a_{2}}^{2}\right\rangle+\left(\alpha_{12}^{*}+\alpha_{21}\right)\left\langle N_{2}\right\rangle\right. \\
& \left.+\frac{1}{2} \alpha_{21}\left\langle N_{1}\right\rangle+\frac{1}{2}\left(\alpha_{12}^{*}+\alpha_{21}\right)\right] \\
& \frac{d}{d t}\left\langle a_{1}^{\dagger 2} a_{2}^{2}\right\rangle=\left[\left(\alpha_{11}^{*}+\alpha_{22}\right)-2\left(\kappa_{1}+\kappa_{2}\right)\right]\left\langle a_{1}^{\dagger 2} a_{2}^{2}\right\rangle+\left[\alpha_{12}^{*}\left\langle a_{1}^{\dagger} a_{2} N_{2}\right\rangle\right. \\
& \left.+\alpha_{21}\left\langle N_{1} a_{1}^{\dagger} a_{2}\right\rangle+\left(\alpha_{12}^{*}+\alpha_{21}\right)\left\langle a_{1}^{\dagger} a_{2}\right\rangle\right], \\
& \frac{d}{d t}\left\langle a_{1}^{2} a_{2}^{\dagger 2}\right\rangle=\left[\left(\alpha_{11}+\alpha_{22}^{*}\right)-2\left(\kappa_{1}+\kappa_{2}\right)\right]\left\langle a_{1}^{2} a_{2}^{\dagger 2}\right\rangle+\left[\alpha_{21}^{*}\left\langle N_{1} a_{1} a_{2}^{\dagger}\right\rangle\right. \\
& \left.+\alpha_{12}\left\langle a_{1} a_{2}^{\dagger} N_{2}\right\rangle+2\left(\alpha_{21}^{*}+\alpha_{12}\right)\left\langle a_{1} a_{2}^{\dagger}\right\rangle\right] \\
& \frac{d}{d t}\left\langle N_{1}^{2}\right\rangle=\left[\left(\alpha_{11}+\alpha_{11}^{*}\right)-4 \kappa_{1}\right]\left\langle N_{1}^{2}\right\rangle+\left[\frac{3}{2}\left(\alpha_{11}+\alpha_{11}^{*}\right)+2 \kappa_{1}\right] \\
& \times\left\langle N_{1}\right\rangle+\frac{1}{2}\left(\alpha_{11}+\alpha_{11}^{*}\right)+\frac{1}{2} \alpha_{12}\left(2\left\langle N_{1} a_{1}^{\dagger} a_{2}\right\rangle-\left\langle a_{1}^{\dagger} a_{2}\right\rangle\right) \\
& +\frac{1}{2} \alpha_{12}^{*}\left(2\left\langle N_{1} a_{1} a_{2}^{\dagger}\right\rangle+\left\langle a_{1} a_{2}^{\dagger}\right\rangle\right), \\
& \frac{d}{d t}\left\langle N_{2}^{2}\right\rangle=\left[\left(\alpha_{22}+\alpha_{22}^{*}\right)-4 \kappa_{2}\right]\left\langle N_{2}^{2}\right\rangle+\left[\frac{3}{2}\left(\alpha_{22}+\alpha_{22}^{*}\right)+2 \kappa_{2}\right] \\
& \times\left\langle N_{2}\right\rangle+\frac{1}{2}\left(\alpha_{22}+\alpha_{22}^{*}\right)+\frac{1}{2} \alpha_{21}^{*}\left(2\left\langle a_{1}^{\dagger} a_{2} N_{2}\right\rangle-\left\langle a_{1}^{\dagger} a_{2}\right\rangle\right) \\
& +\frac{1}{2} \alpha_{21}\left(2\left\langle a_{1} a_{2}^{\dagger} N_{2}\right\rangle+\left\langle a_{1} a_{2}^{\dagger}\right\rangle\right),
\end{aligned}
$$

where the coefficients $\alpha_{11}, \alpha_{22}, \alpha_{12}$, and $\alpha_{21}$ are listed in Appendix B.

\section{APPENDIX B}

Considering that all atomic levels decay at the same rate $\Gamma$ and taking $\Delta_{1}=\Delta_{2}=\Delta$, the coefficients $\alpha_{11}, \alpha_{12}, \alpha_{21}$, and $\alpha_{22}$ in Eq. (7) are given by the following:

$$
\alpha_{11}=\frac{g^{2} r}{D D^{\prime}}\left\{\frac{1}{\Gamma} G_{21} \Omega_{1}^{2}\left[2\left(\Gamma+2 Z_{d}\right) Z_{d}+\Omega_{2}^{2}\right]-\Omega_{1}^{2} \Omega_{2}^{2} \Omega_{D}\right\},
$$

$$
\begin{gathered}
\alpha_{22}=\left.\alpha_{11}\right|_{1 \leftrightarrow 2}, \\
\alpha_{12}=\frac{g^{2} r}{D D^{\prime}}\left\{\frac{1}{\Gamma} G_{12} \Omega_{1} \Omega_{2}\left[2 \Gamma Z_{d}-\Omega_{2}^{2}\right]+\left(4 Z_{D}^{2}\right.\right. \\
\left.\left.+\Omega_{2}^{2}\right) \Omega_{1} \Omega_{2} \Omega_{D}\right\}, \\
\alpha_{21}=\left.\alpha_{12}\right|_{1 \leftrightarrow 2},
\end{gathered}
$$

with

$$
\begin{gathered}
G_{12}=2 \Gamma\left(2 \Gamma^{2}+\Omega_{1}^{2}\right)+\Gamma\left(\Omega_{2}^{2}-\Omega_{1}^{2}\right), \\
G_{21}=\left.G_{12}\right|_{1 \leftrightarrow 2} \\
\Omega_{D}=4 \Gamma^{2}+\Omega_{1}^{2}+\Omega_{2}^{2}, \\
D=4 \Gamma^{5}+\Gamma^{2}(\Gamma+4 \Gamma)\left(\Omega_{1}^{2}+\Omega_{2}^{2}\right)+\Gamma\left(\Omega_{1}^{4}-\Omega_{1}^{2} \Omega_{2}^{2}+\Omega_{2}^{4}\right) \\
+3 \Gamma \Omega_{1}^{2} \Omega_{2}^{2}, \\
D^{\prime}=Z_{D}\left(4 Z_{D}^{2}+\Omega_{1}^{2}+\Omega_{2}^{2}\right), \\
Z_{D}=\Gamma+i \Delta .
\end{gathered}
$$

[1] H. Xiong, M. O. Scully, and M. S. Zubairy, Phys. Rev. Lett. 94, 023601 (2005).

[2] H. T. Tan, S. Y. Zhu, and M. S. Zubairy, Phys. Rev. A 72, 022305 (2005).

[3] L. Zhou, H. Xiong, and M. S. Zubairy, Phys. Rev. A 74, 022321 (2006).

[4] S. Qamar, H. Xiong, and M. S. Zubairy, Phys. Rev. A 75, 062305 (2007).

[5] M. O. Scully and M. S. Zubairy, Phys. Rev. A 35, 752 (1987); in some earlier studies, the experimental observation of the noise reduction in the relative phase of a two-mode laser has also been discussed; see M. Ohtsu and K.-Y. Liou, Appl. Phys. Lett. 52, 10 (1988); M. P. Winters, J. L. Hall, and P. E. Toschek, Phys. Rev. Lett. 65, 3116 (1990).

[6] M. Ikram, G.-X. Li, and M. S. Zubairy, Phys. Rev. A 76, 042317 (2007).

[7] S. Qamar, F. Ghafoor, M. Hillery, and M. S. Zubairy, Phys.
Rev. A 77, 062308 (2008).

[8] J. Hald, J. L. Sorensen, C. Schori, and E. S. Polzik, Phys. Rev. Lett. 83, 1319 (1999); B. Julsgaard, A. Kozhekin, and E. S. Polzik, Nature (London) 413, 400 (2001).

[9] Y. Zhang, H. Wang, X. Y. Li, J. T. Jing, C. D. Xie, and K. C. Peng, Phys. Rev. A 62, 023813 (2000).

[10] C. Silberhorn, P. K. Lam, O. Weiss, F. Konig, N. Korolkova, and G. Leuchs, Phys. Rev. Lett. 86, 4267 (2001).

[11] W. P. Bowen, N. Treps, R. Schnabel, and P. K. Lam, Phys. Rev. Lett. 89, 253601 (2002).

[12] C. Simon and D. Bouwmeester, Phys. Rev. Lett. 91, 053601 (2003).

[13] A. S. Villar, L. S. Cruz, K. N. Cassemiro, M. Martinelli, and P. Nussenzveig, Phys. Rev. Lett. 95, 243603 (2005).

[14] K. Ahmed, H. Xiong, and M. S. Zubairy, Opt. Commun. 262, 129 (2006).

[15] S. Y. Lee, S. Qamar, H. W. Lee, and M. S. Zubairy, J. Phys. B 
41, 145504 (2008).

[16] S. Qamar, M. Al-Amri, and M. S. Zubairy, Phys. Rev. A 79, 013831 (2009); 79, 039902(E) (2009).

[17] U. W. Rathe and M. O. Scully, Phys. Rev. A 52, 3193 (1995).

[18] See M. Hillery and M. S. Zubairy, Phys. Rev. Lett. 96, 050503 (2006), and references therein.

[19] R. Simon, Phys. Rev. Lett. 84, 2726 (2000); L. M. Duan, G. Giedke, J. I. Cirac, and P. Zoller, ibid. 84, 2722 (2000).

[20] H. P. Yuen and J. H. Shapiro, IEEE Trans. Inf. Theory 26, 78 (1980).

[21] S. Qamar, S.-Y. Zhu, and M. S. Zubairy, Opt. Commun. 147, 274 (1998).

[22] C. H. Bennett, G. Brassard, C. Crepeau, R. Jozsa, A. Peres, and W. K. Wootters, Phys. Rev. Lett. 70, 1895 (1993).
[23] C. H. Bennett and S. J. Wiesner, Phys. Rev. Lett. 69, 2881 (1992).

[24] A. K. Ekert, Phys. Rev. Lett. 67, 661 (1991).

[25] A. Barenco, D. Deutsch, A. Ekert, and R. Jozsa, Phys. Rev. Lett. 74, 4083 (1995).

[26] S. L. Braunstein and P. van Look, Rev. Mod. Phys. 77, 513 (2005).

[27] D. Meschede, H. Walther, and G. Muller, Phys. Rev. Lett. 54, 551 (1985); G. Raqithel, C. Wagner, H. Walther, L. M. Narducci, and M. O. Scully, in Advances in Atomic Molecular and Optical Physics, edited by P. Berman (Academic, New York, 1994), Supp. 2, p. 57.

[28] J. M. Raimond, M. Brune, and S. Haroche, Rev. Mod. Phys. 73, 565 (2001). 